\title{
Openness in Computing The Case of Linux for Translators
}

Peter Sandrini

University of Innsbruck, Austria

The decision to use exclusively open source software for translation purposes includes deploying an open source operating system. Put in another way, if I, as a translator, want to use free and open source applications on my PC, it is legitimate and almost obvious for me to support this choice by using an open source operating system as well. An operating system constitutes the basic infrastructure of any computer system: without it, no application can be launched and no data can be edited or saved.

In this context, openness first and foremost means using a free and open source operating system, thus, eliminating the need for any proprietary software; secondly, openness is also about having the opportunity to be part of a community, by sharing and contributing one's own experiences and solutions.

The following paper describes the use of GNU/Linux as a platform for translation, summarizes experiences and opportunities, and gives a historical overview over different initiatives trying to adapt the GNU/Linux environment for translation.

\section{$1 \mathrm{GNU/Linux} \mathrm{-} \mathrm{The} \mathrm{Operating} \mathrm{System}$}

GNU/Linux is a piece of software "that enables applications and the computer operator to access the devices on the computer to perform desired functions" (Linux Foundation 2015). It represents the deep software layer of a computer systems on which all other applications build upon. What sets GNU/Linux apart from comparable commercial solutions, such as Microsoft's Windows or Apple's OS $X$, is the collaborative development based on a community of programmers who contribute to the system. Nobody owns GNU/Linux and there is no single company responsible for GNU/Linux even though a few commercial companies contribute code on a regular basis; there are, however, numerous communities, each working on a specific component of the system.

The story of GNU/Linux begins when in the late 1970s a programmer at MIT, Richard Stallman, became dissatisfied with the increasing commercialization of the old UNIX computer operating environment. He began to develop a set of tools, called the GNU (GNU Is Not Unix) tools, as a first step 
on the way to a free operating system. While the main tool-set was ready rather quickly, the central part of the operating system, called its kernel, was still missing and the corresponding HURD kernel project lagging behind time. In 1991 the Finn programmer Linus Torvalds programmed a new kernel and gave it the name Linux. Thus, the Linux kernel successfully complemented the GNU tools and became the core architecture of a complete and open source operating system, the GNU/Linux system (Stallman 2014).

There are general arguments in favor of GNU/Linux over other OSs: over the years, it has become a stable and mature operating system which can easily replace any other system. A strong emphasis on security, for example, makes anti-virus software more or less obsolete, a robust system architecture avoids frequent rebooting, thus increasing efficiency and productivity.

These general advantages, however, may not be the main reason for a change to GNU/Linux; it is its openness and free availability, giving the user a choice of more than 500 different flavors of Linux distributions. GNU/Linux relies on the work of communities, it is free software and as such it is subject to the four essential freedoms as defined by the Free Software Foundation (outlined in the introduction to this volume). With these freedoms, the users, both individually and collectively, gain control over their computers and the technology they use:

- Users can be assured that their computing remains confidential as the code is open and back-door attacks to the system are immediately detected and removed.

- The integrity of the program code is guaranteed through its openness.

- The integrity of user data is guaranteed through the stable system architecture and the almost complete absence of viruses.

- Users have complete freedom over installation and configuration of software.

- Users have a choice and can be part of a community, changing from dependent consumers of a purchased product into active and autonomous agents, completely independent of commercial interests and big companies.

The advantages of having full control over one's own PC includes ease of computer installations without having to input activation codes or managing software licenses. Still, there is no fear of copyright infringements even when multiple instances of the system are installed, e.g. on a desktop and a notebook computer, or in a computer lab of a school or university. For students and university graduates full control also allows a cost-saving start of 
their professional career which is especially important during a first orientation period.

Openness and control over the computer system also facilitates co operation with colleagues by eliminating the risk of malware and viruses, by supporting open standards, as well as by fostering discussion and exchange through participation in on-line communities in support of free and open source projects.

\subsection{Language Support}

In addition to having control over their own computer, users may count on a rather extensive language support, in many cases exceeding that of commercial operating systems. The mainstream GNU/Linux distribution Ubuntu, for example, supports around 150 languages: it comes in English by default, but users may choose from more than 146 additional languages to install, and get the user interface in their mother tongue. This originates from the fact that GNU/Linux developers are organized in many individual projects scattered all over the world, so that language support even for smaller and less developed locales was recognized as a necessity right from the beginning. For this purpose, a thorough localization method has been introduced for the operating system as well as for all applications meant to run on it: the GNU GETTEXT environment, designed to minimize the impact of internationalization and localization on the program source code.

Specifically, the GNU GETTEXT utilities are a set of tools that provide a framework within which free software packages can produce multilingual messages, as well as a set of conventions about how programs should be written to support message catalogs. These message catalogs, called PO files, contain both the English and the translated versions of each message. $\mathrm{PO}$ stands for Portable Object, distinguishing it from MO files or Machine Object files. PO files are meant to be read and edited by humans, and associate each original, translatable string of a given package with its translation in a particular target language. PO files are strictly bilingual, as each file is dedicated to a specific target language. If an application supports more than one language, there is one such PO file per language supported.

The utility program XGETTEXT creates a PO Template file (POT) by extracting all marked messages from the program code sources, the MSGINIT tool converts it into a human readable PO file. Another utility, MSGMERGE, takes care of adjusting PO files between releases of the corresponding sources, excluding obsolete entries, initializing new ones, and updating all source line references. Translators then edit and translate the 
messages contained in the files with the help of simple text editors or dedicated PO file editors such as Lokalize, the PO file editor of the KDE desktop environment, Gtranslator and PO-Edit from the GNOME desktop environment, or PO Mode, a specific add-on for the text editor Emacs. PO files are only used as an intermediate file format in the development and localization process: after translation, the MSGFMT tool converts PO files to binary resource files, or MO files, which are then used by the GETTEXT library at run time.

The GNU GETTEXT environment was one of the first thorough software localization methods and it was introduced by the free software community and the GNU/Linux system in 1995. PO files also constituted the first translation data format long before XML formats such as TMX and XLIFF were invented. The localization of free and open source programs is well supported and documented; the excellent introduction written as a Master thesis by Arjona Reina (2012) explains the process in detail and gives an overview over tools and platforms.

Once translations are in place, users can influence the language used by the operating system and by installed applications in different ways:

1. During the installation of the system, users may choose a preferred language which sets the system-wide default language for all users, as well as the language used when a new user account is created; each user can have his own locale configuration that is different from the locales of the other users on the same machine.

2. By setting the GUI language of a desktop environment, such as KDE, GNOME, or XFCE, which usually includes the window manager, a web browser, a text editor, and other applications. The locale used by GUI programs of the desktop environment can be specified in a special configuration screen.

3. By configuring a series of environment variables like LANGUAGE, LC_ALL, LC_XXX, LANG.

In addition, text input can be adapted to different writing systems by installing specific tools and setting up the operating system accordingly. Furthermore, Unicode, the Universal Character Set standard, capable of encoding, representing, and handling of text expressed in most of the world's writing systems, has become standard in most GNU/Linux installations.

Because of the GNU GETTEXT environment and the versatility of configuration options, modern GNU/Linux distributions are indeed well suited as multilingual computer systems for everybody who needs to use, write or work with two or more languages. 


\subsection{Adoption}

Today, most users who face a GNU/Linux system for the first time already had some experiences with a proprietary operating system. A change of the main operating system involves a certain degree of readjustment: new interface, new commands, new system applications and a new way of organization have to be learned. The whole change may be represented as "trading Windows problems for Linux challenges" (Hartley 2015). GNU/Linux is not more difficult to handle than other proprietary systems (see survey results in García González 2013: 141) as it has often been blamed, it is just different, and users have to adjust. This initial difficulty is often mistaken for greater complexity, but it is not, as GNU/Linux users who return to using a proprietary system, very often encounter the same challenge.

GNU/Linux comes in a variety of distributions, each one with its particular features, some even geared to a specific task. The main distinction to be made, however, is the discrimination of three specific areas of use: as a server operating system, a desktop system or a mobile operating system. While GNU/Linux on servers has a share of $36 \%$ for public servers on the Internet and 97\% for supercomputers in 2015 according to Gartner research (Wikipedia n.d.), and Linux on mobile devices, including Android which uses the Linux kernel, tops all other operating systems, it struggles to achieve the same results on the desktop. Adoption rates on desktop systems are very hard to get and in most cases the operating system is identified by web counters. The figures coming from such web counters attribute a rather small market share to GNU/Linux: from 1.47\% for 2015 (Net Market Share n.d.) to around 5\% (W3schools n.d.). The Linux Counter Project website (Linux Counter Project n.d.) describes the difficulties in assessing exact numbers of users, but estimates the number of GNU/Linux users worldwide at $79,879,362$.

The number of users of specialized GNU/Linux distributions, such as the distributions for translators mentioned below, are even harder to assess: there are numbers of downloads, e.g. from Mediafire where tuxtrans is hosted, or the number of participants and messages in on-line discussion groups, but they all only indicate trends, show interest, but they do not give evidence of the number of actual users. In view of available numbers, even if these data is highly unreliable, we have to conclude that, basically, GNU/Linux remains a niche operating system on the desktop, and, thus, also in the translators community.

However, several initiatives and projects have recognized the advantages and usefulness of free and open source software in general, and on the desktop in particular. The European Union's Open Source Software Strategy 
2014-2017, for example, states that the EU "Commission shall continue to adopt formally, through the Product Management procedure, the use of OSS technologies and products", in order to "ensure a level playing field for open source software and demonstrate an active and fair consideration of using open source software" (EU Commission 2015: art 1 and 2). For this purpose, several initiatives were launched within the EU, e. g. the Joinup collaborative platform (EU Commission 2015b) aiming at interoperability solutions for public administrations, formerly called OSOR, the Open Source Observatory.

\section{GNU/Linux for Translators}

When we speak of a free operating system for translation and translators, we need to specify this particular target group more clearly. Translators may be single free-lance translators, working on a desktop computer, they may be translating voluntarily in their spare time for non-governmental organizations, open source software or charity projects, or they may work for a translation agency as professional translators to earn a living. In today's globalized world, all translators rely on some form of networking or Internet use, be it the exchange of translation memories or other language data between voluntary translators, the use of on-line or cloud-based translation tools, such as Google Translator Toolkit (GTK n.d.), Dotsub, a cloud-based subtitling platform (Dotsub n.d.), the Trommons, a web-based translation environment developed by The Rosetta Foundation (The Rosetta Foundation n.d.), or even the use of on-line translation memories tools such as Matecat (Matecat n.d.), Linguee (Linguee n.d.) or MyMemory (MyMemory n.d.) or on-line term banks. Networking and Internet use, however, become a necessity for professional translators for which cooperation and on-line presence is a must: Cronin speaks of the network-based nature of the translation industry "where translation projects are managed across countries, continents, cultures and languages" (Cronin 2003: 45).

Translators are, thus, a diverse target group and translation is far from a homogeneous activity. Yet, some common features and prerequisites for a computer system suitable to the task of multilingual communication and translation may be identified:

- Wide multilingual support - language support comprises a wide choice of languages for the user interface of the OS, language support for installed applications, support for different text input systems and language-specific keyboards layouts. 
- Support for standards, especially standards regarding multilingual text (Unicode n.d.), writing systems (text input, fonts), translation (PO, TMX, XLIFF), terminology (TBX).

- Inclusion of translation technology applications: CAT, MT, TM, Terminology, etc.

For a translation-oriented computer system, specific applications must be included, configured and installed. The operating system only represents a platform for applications of translation technology, and translation technology applications run on such a platform. This creates a mutual dependency: an operating system for which no specific translations-oriented applications exist is of no use, and software applications will not work without the basic infrastructure of the operating system.

Technology has become indispensable in many areas, for translation scholars even speak of a "technological turn" (Cronin 2010: 6). Translation technology has been around more or less thirty years now, but the number of available software products as well as specific free and open source projects has multiplied in recent years. Translation technology may be defined as any kind of digital Information and Communication Technology (ICT) which supports or performs the translation process with the aim of meeting adequate efficiency and quality requirements (Sandrini 2012: 111). While for many translators, translation technology still equals Trados, a widely used proprietary CAT tool, a number of specific translation-oriented applications have been developed exclusively for or ported to the Linux environment, so that today there is a variety of options available. Commercial products, such as Swordfish, Wordfast Pro, Cafetran, XTM, MemSource and others, are available on the market on the basis of proprietary licenses, and more importantly, a plethora of free and open source software applications is listed in the FOSS4Trans catalog (FOSS4Trans n.d.) with no less than 150 specific programs for GNU/Linux subdivided into four broad categories:

37 editing and publishing tools (plain text and code editors, office suites, desktop publishing, advanced image editors, subtitle editors, optical character recognition software, differencing tools, PDF tools);

30 language tools (terminology extraction, text analysis, corpus creation and processing, resource lookup tools, language checkers);

59 translation tools (translation environments, machine translation programs, localization tools, alignment tools, format conversion and validation utilities for translation-related formats);

24 management tools (project management programs, word-counting and invoicing tools, financial management software, reference management tools, quality assurance tools). 
Although some items in the list do not strictly qualify as translation tools proper, and some software projects have already stopped development, this catalog is good evidence of the availability of translation technology applications for the GNU/Linux platform. Thus, the argument often brought forward against GNU/Linux that there are too few CAT tools for this platform is no longer valid.

There is, however, one main difference between some proprietary systems and GNU/Linux, or more in general, Unix. Applications developed for this platform mainly follow a specific design principle which goes "make each program do one thing well" (Mcllroy et al 1978: 1902), as outlined early by its main programmers. Programs are designed to do one thing and do it well, so that applications focus on just one specific task. As a consequence, in the free and open source community we have a great number of individual projects creating applications with specific functionality on the basis of this principle: one or more communities developing a terminology management system, others developing terminology extraction tools or terminological/lexicographical file format converters, projects dedicated to spell checking routines, communities developing text format conversion tools, others creating translation project management programs, yet others implementing accounting software for translators, and so on. This splitting-up of human resources is somewhat attenuated by a second GNU/Linux and Unix programming principle which says: "write programs to work together" (Mcllroy et al 1978: 1902), making the output of every program the potential input to another program. Communication and data exchange between programs, thus, becomes crucial and of central importance. So, you may end up with many different tools but they are all able to interconnect in one way or another.

Opposed to a great number of translation technology applications in the free and open source world each of which concentrating on one specific functionality, we find huge all-encompassing software programs, called TransIation Environment Tools (TEnT) (Zetzsche 2014: 189) in the proprietary and commercial world. Such a computer-aided-translation tool or TEnT aims at providing a one-stop solution for translators with all needed functionality, from a translation-memory engine, terminology management, alignment, collocation search and translation project management, up to format conversion, spell-checking, text editing and formating tools, etc. This results in having only a few contenders for market leadership in the commercial environment, but a great number of projects and communities in the free and open source world. Translators exploring free and open source programs should get accustomed to the thought that there is more than one program for a specific task and that they are expected to try out and combine different applications for a useful translation workflow. 
Translation Technology can boost the efficiency and consistency of translation, but inconsiderate use of software and services may also cause translators losing control over the translation process and translation data. This is especially true for web-based translation tools, software-as-a-service applications (SAAS) and closed source programs. Costs and risks of software technology should always be evaluated: this could give free and open source tools a clear advantage due to their very low access barrier with regard to costs, and their security and reliability.

In combination with the principle of openness, the specific features and advantages of the GNU/Linux operating system may be summed up as follows:

- a stable and secure operating system

- good usability, easy handling and configuration

- full control over the system

- variety of available FOSS applications

- no financial costs, free to use and free to redistribute.

A change to GNU/Linux, however, always involves a certain degree of rethinking one's habits and practices using the computer, it demands adaptation, and could be a learning challenge. On the other side, such a change opens up a new world of unfettered use of the computer and, according to the Distrowatch.com website, it puts "the fun back into computing" (Distrowatch.com n.d.).

\subsection{Linux Distributions for Translation}

Acknowledging these advantages, a few initiatives promoting the use of GNU/Linux in translation were launched in the last two decades. One of the first initiatives was a website (Prior 2010) created by Marc Prior around the year 2000 in which he reports on his experiences using GNU/Linux as a translator in his day-to-day work. He lists and describes applications of interest to translators, shares his experiences and offers links to many GNU/Linuxrelated websites. Hand in hand with this website a discussion group was created on Yahoo, the Linux for Translators Forum, "intended primarily for professional translators who use GNU/Linux software for their work" (The Linux for Translators Forum 2002: About Group) with 614 members at the time of writing. Discussions in this group address all topics regarding the use of GNU/Linux for professional translation tasks. 


\section{Linux for Translators Forum: $\mathrm{n}^{\circ}$ of messages}

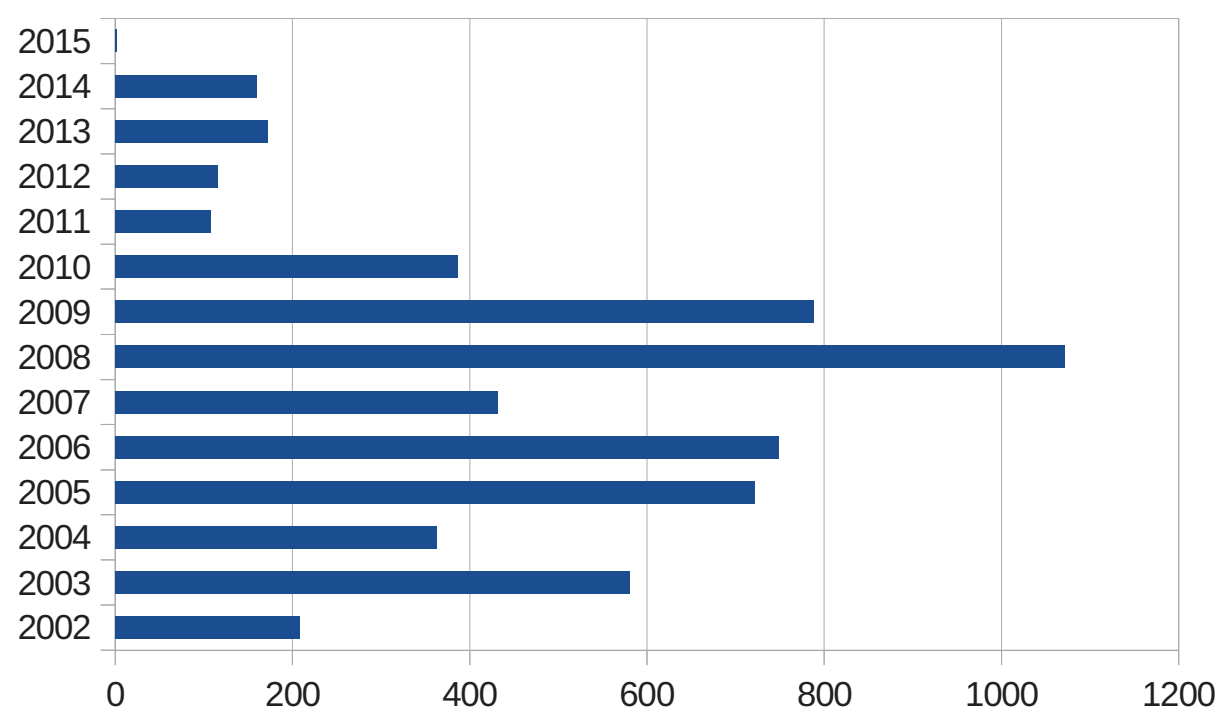

Figure 1: Number of messages in the Linux for Translators Forum.

Figure 1 shows that interest in this forum peaked in 2008 with more than 1000 messages, and settled down to around 150 messages a year after 2011. The time around 2007 and 2008 also was the beginning of dedicated GNU/Linux distributions for translators while the first years of the millennium saw the development of some of the most prominent free and open source applications in translation like OmegaT, Pootle, Open Language Tools, Apertium, Moses, Globalsight and others.

The use of GNU/Linux has also been the topic of discussions in user forums or websites dedicated to professional translation such as ProZ.com and Translatorscafe.com. Starting from 2005, the "Festival Latinoamericano de Instalación de Software Libre" (FLIsoL n.d.), a series of regular events in Latin America, promotes the use of free software and free culture, organizing among other things workshops about the use of Ubuntu and tuxtrans for students.

In 2007, the group GETLT (Grupo de Estudos das Tecnoloxías Libres da Tradución (GETLT n.d.) was created at the University of Vigo, Spain, with the following goals in mind: to analyze and promote the use of free software in professional translation practice, as well as in translator training; promote the 
visibility of the work done by volunteer translators of free projects; stimulating the cooperation of students, teachers and professionals in translation with communities involved in translating free software projects. In addition to relevant publications (Diaz Fouces et al. 2008, Diaz Fouces 2010, García González 2013), the main product of this group was the development of a translation-oriented GNU/Linux distribution called MinTrad.

Distributions are software packages which include GNU/Linux, as well as a number of selected applications. A GNU/Linux system, with its set of tools surrounding the kernel, different window managers and a great number of complete desktop environments providing the graphical user interface (GUI) and allowing the interaction with the user, is very modular, and for each component, numerous projects have developed slightly or totally different compatible versions which may be exchanged at the discretion of the user. Due to this modularity, a GNU/Linux system may be configured and set up in many different ways, for different tasks and different environments. This generated several independent releases of GNU/Linux called distributions Distrowatch.com lists more than 500 of them - where the distribution's makers which may be a company, an individual or a community have decided which kernel, operating system tools, environments, and applications to include and ship to users.

A few attempts have been made to tailor a GNU/Linux system to the requirements of a translator, making choices with regard to two different aspects: 1) decision about which system tools, window managers or desktop environments to include, and 2) decision about what applications to configure and install. Ideally, both decisions should be based upon how well multilingual support, open standards and translation technology are supported; but in some cases, e. g. the choice of a desktop environment like KDE, Gnome or XFCE, it may be a matter of personal preferences.

The following GNU/Linux distributions have been developed explicitly for translators and include free and open source standard applications like web browsers, email clients, office suits - mostly LibreOffice -, as well as dedicated translation technology software, such as translation memory systems mostly OmegaT -, terminology applications and text analysis programs. These categories are the most commonly used applications by professional translators (see survey results in García González 2013: 137).

\section{Linguasos}

LinguasOS was developed by Tony Baldwin, a "translator and translation agency owner who is intimately familiar with the needs of professionals in the translation trade" (Baldwin 2008) in December 2007. It is a based on 
PCLinuxOS, more specifically on PCFluxboxOS with the minimalistic window manager Fluxbox, and adapted for professional translators and those working in software localization with many specific applications and support for all industry standard file formats.

LinguasOS "a) attempts to give translators a platform for experimenting with the tools that are available in FOSS for the trade, in a quick and light Live CD distribution, as well as, b) provides an easily maintained, preconfigured OS for translators that are already using, or wish to begin using Linux for their work" (Baldwin 2008).

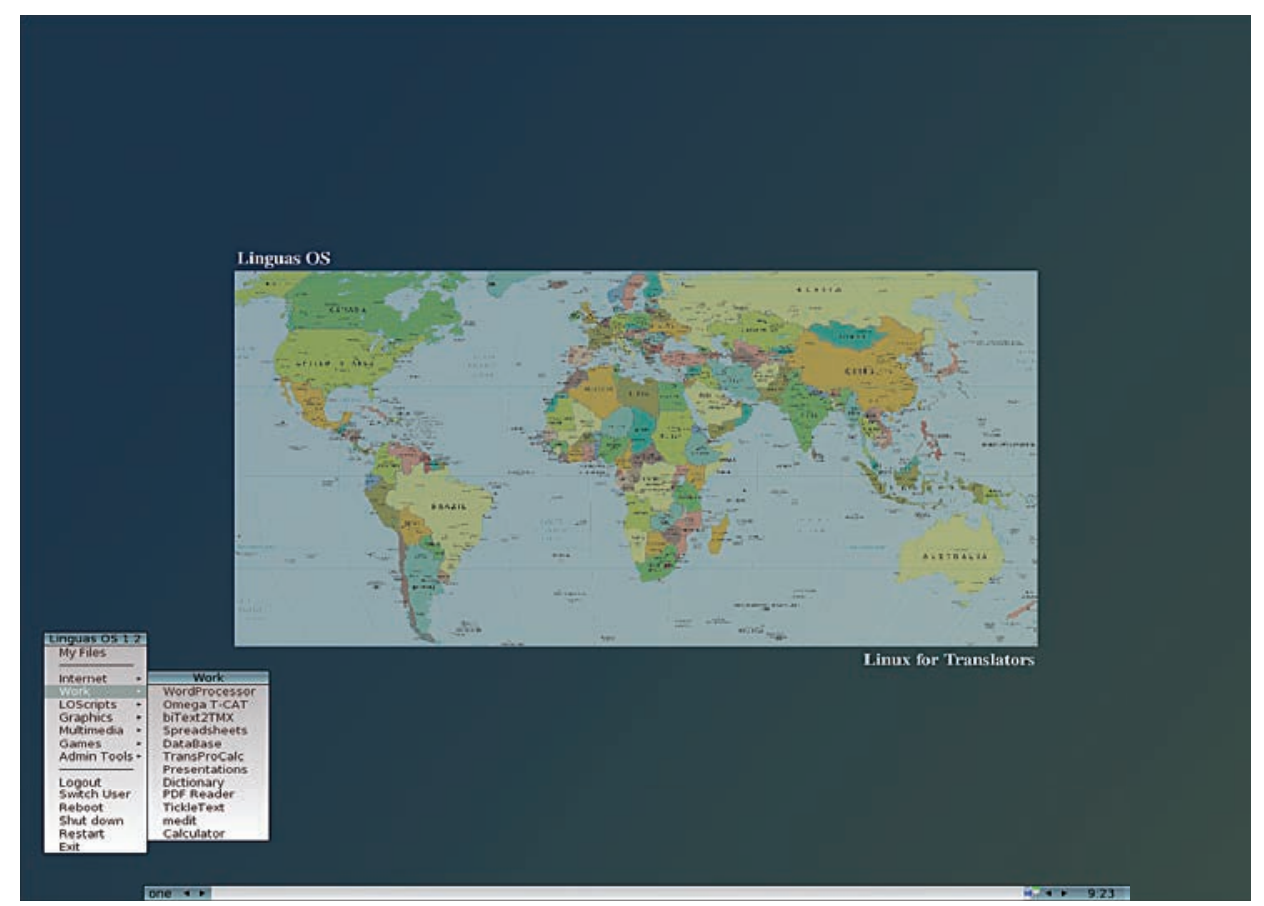

Figure 2: LinguasOS start screen and application menu.

The system comes as a live-CD packaged in an ISO file with only $412 \mathrm{MB}$ of disk space which can be started for trial purposes from a CD or a USB stick without installing or changing anything on the computer; however, installation on the hard-disk is also possible.

The user forum (LinguasOS discussion group n.d.) has messages going from December 2007 through February 2010. LinguasOS is still listed on 
Distrowatch.com with its most recent release (1.3) dated March 2008, though development was officially stopped in October 2009.

\section{MinTrad}

From 2007 to 2010 the GETLT group at the University of Vigo in Spain launched a project with the title "Creation of a GNU/Linux training environment for the training of translators, software localizers and subtitle editors" (see García González 2013: 130 and Veiga Díaz/García González in this volume) financed by the Galician Regional Government, with a slightly different target group focusing on academic teaching, and widening the concept of translators to include multilingual communication and localization. The resulting distribution MinTrad is based on Linux Mint and features a traditional desktop with a custom menu item 'MinTrad' listing all translation-specific programs.

The Linux Mint basis represents a user-friendly and reliable system and the choice of programs is well thought-out, even though OmegaT comes in three slightly different versions (OmegaT, Autshumato, OmegaT+). However, the last version in the download section of the FTP-server (ftp.uvigo.es/mintrad/) accessed at the time of writing, is dated September 2012.

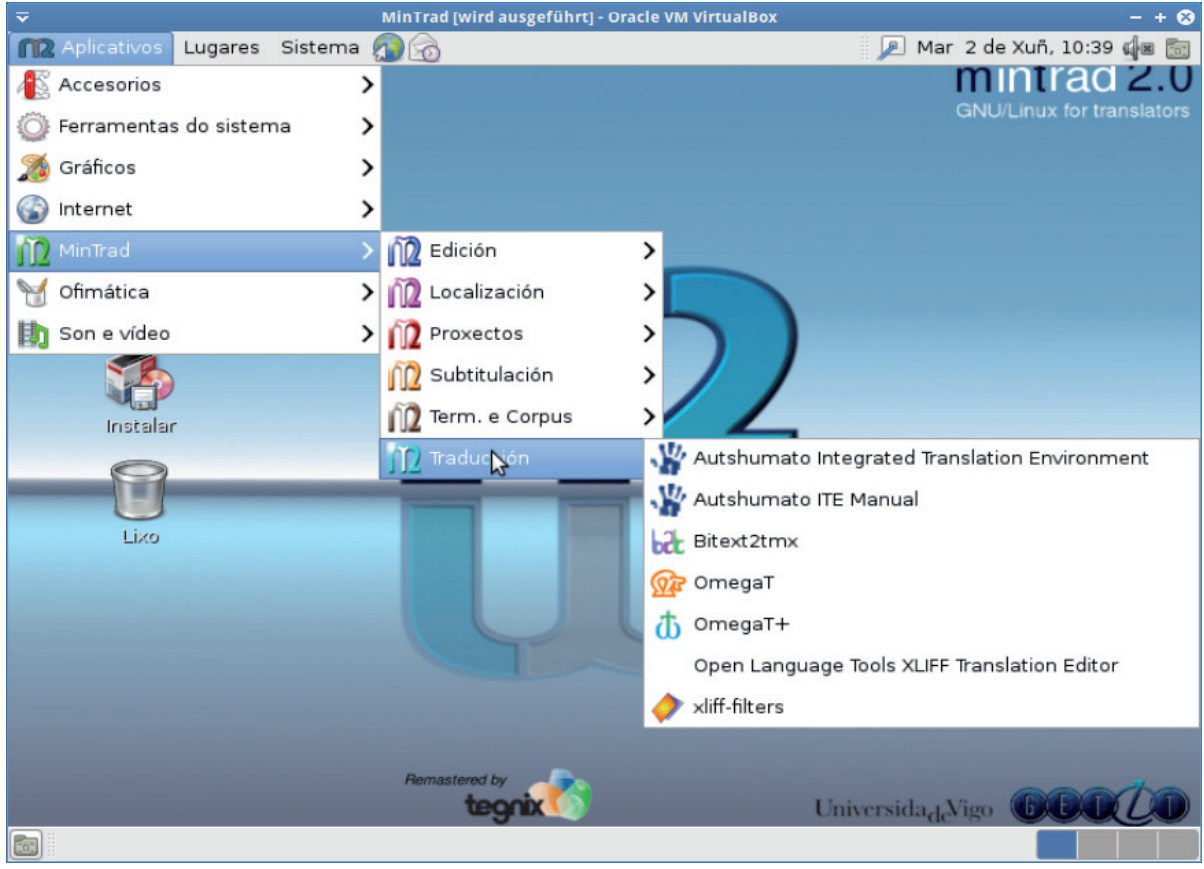

Figure 3: MinTrad start screen and application menu. 


\section{tuxtrans}

More or less at the same time, in December 2007, another customized system for translators called PCLOSTrans was created at the University of Innsbruck in Austria. It was based on PCLinuxOS a more general GNU/Linux distribution featuring the KDE desktop; in 2010 this basis was exchanged for the widely used Ubuntu distribution with both the XFCE and the Fluxbox desktop and the name was changed to tuxtrans. The most important open source applications of translation technology are included and made accessible through the customized menu 'Translation'. The user interface is available in four languages English, Italian, German and Spanish, but more may be installed on-line from the Ubuntu repositories.

A dedicated user forum (tuxtrans discussion group n.d.) lists messages going from May 2010 through January 2015, and the tuxtrans website (tuxtrans n.d.) has introductory notes on how to install and use the system, as well as a FAQ page. The system comes in a 32bit and a 64bit version and the last update available for download is dated September 2014 (32bit) and March 2015 (64bit).

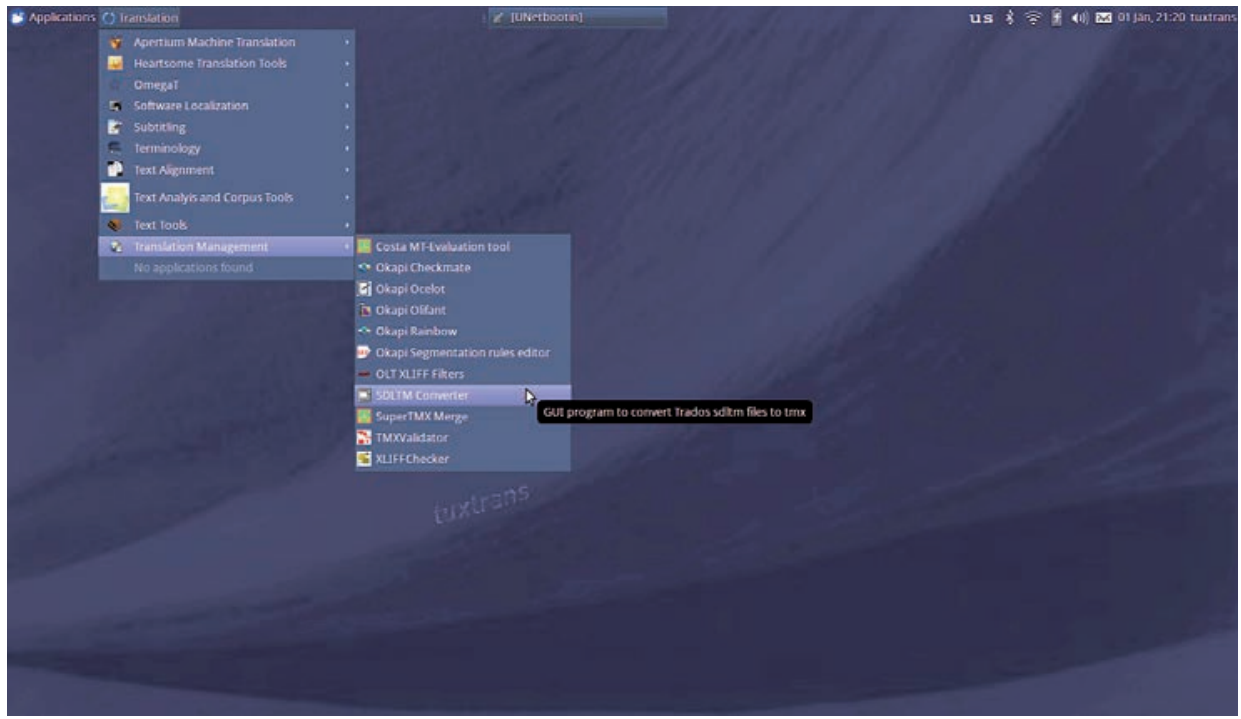

Figure 4: tuxtrans start screen and application menu.

Apart from standard applications and the most common translation technology programs, the three distributions differ in their integration of machine translation and locally installed web-based applications. Every 
GNU/Linux system can act as a Web server if the right software is installed. Thus, it would be an interesting future path to move from a single-user desktop system to a distribution which already includes the necessary infrastructure software (e.g. databases, web servers) coupled with multi-user translation technology applications, such as for example, the translation management system Globalsight, the terminology management system Autshumato TMS, a multilingual web content management system like Drupal, a translation server like Pootle, etc. Such a GNU/Linux system can be used either as desktop system or, when properly installed, as a multi-user translation server, or with the appropriate hardware, even both uses at the same time on the same machine are possible.

For machine translation, there is already Apertium working off-line which can be installed very easily as a Java application with all the language combinations supported. The Moses MT system, another open source machine translation system, requires much more effort and know-how for installation, and, in particular, plenty of disk space for a working instance with one language combination, and each new language combination adds further disk space; installation of such a language-specific, or better language-combination-specific program in a general, translation-oriented distribution, therefore, does not make much sense without a limitation to two working languages.

\section{Conclusion}

Even though two of the three distributions are not updated any longer, these projects still prove that using exclusively free and open source software does constitute a real option for any kind of translator, allowing her to do all relevant tasks in translation and localization. Nonetheless, there is still no sign of a wide adoption of GNU/Linux as an operating system for translators, and no major breakthrough has been made, at least judging from GNU/Linux adoption in general, and direct feedback, questions and reports from users of tuxtrans, in particular.

With all the advantages mentioned earlier, the robustness of the system, the possibility of easy testing with live-systems booting from a DVD or a USB stick, and, last but not least, the negligible cost, the question has to be raised what factors prevent users in translation, localization or multilingual communication from adopting GNU/Linux. A few possible reasons can be tentatively mentioned:

- Reluctance to change to a new operating system from the old accustomed one; in many cases, new computers come with a preinstalled proprietary operating system, and in many companies or 
institutions only one proprietary system is supported, so that users usually start out with this system and get used to it, thus increasing the barriers for a change;

- Assumed or real complexity of GNU/Linux;

- Absence of professional support; with GNU/Linux there is nobody to blame when something goes wrong, except one's own knowledge and preparation. For some users the change from commercial support to voluntary support through communities may pose a challenge;

- Incompatibility of specialized software; not all software programs run under GNU/Linux. That being said, the best approach would be to look for comparable (functionality) and compatible (support for standards) applications, i. e. when a user says "I cannot use GNU/Linux because Trados does not run on it", the right question to ask would be "What are the reasons for using Trados?" and "Could the free translation memory system OmegaT replace it?" as well as "Can you exchange translation memory files on the basis of TMX?" In many cases this could solve the problem, provided there are no other more serious reasons;

- Lack of awareness and not knowing about GNU/Linux and free and open source options: this is, among other things, why this article is written. Poor knowledge about GNU/Linux and free and open source software in general among translators and translation students has been mentioned in a survey conducted in 2008 (García González 2013): "the almost complete unawareness of the characteristics and possibilities of open-source software revealed by the participants" (García González 2013: 136).

All this reasons could deter translators from using GNU/Linux, but it is nearly impossible to identify the most important, or the most influential factors. Instead of guessing what keeps users/translators from using GNU/Linux, maybe everyone, or every computer user should better ask: Why should I not use a free and open source operating system that is freely available, secure, multilingual and ready to be used for translation? And: Why should I, then, pay for a proprietary operating system?

With no clear picture about the key factors influencing user adoption, it could be useful to identify common measures that address all of these factors. Intervening on the last one, i. e. to inform and educate potential users about free and open source software and operating systems, seems to be also at the heart of the other reasons where a lack of knowledge or understanding constitutes a major problem. This is done primarily through public promotions by non-governmental organizations, like the Free Software Foundation (FSF 
n.d.), the Free Software Foundation Europe (FSFE n.d.), and others, or by personal initiatives.

Promoting and enhancing information and awareness about free and open source software could be done best within academic organizations and translator training institutions where future translators learn about the choices and options they have when it comes to translation technology and basic IT infrastructure. Narrowing down their options to proprietary systems would not be in accordance with good academic practice: teaching at university level is, indeed, more about empowerment of students than simple product training (Diaz Fouces 2011). All the advantages of using free software in education (FSFE n.d.) apply to the use of a free and open source operating system as well: no license fees, no trouble with licenses, equality for all students and teachers, etc, in addition to the general advantages mentioned earlier in this contribution.

With this in mind, we may answer the question why the makers of specific distribution of GNU/Linux for translators are doing this work and providing such a system for free. From personal experience, I would say, they do it as prove of practicability, because it can be done, or even because somebody needs it. In the case of tuxtrans, the fact that it actually represents the system I am working with myself, greatly facilitates the production of this distribution. Independently of the number of potential users, free software allows me to make my desktop computer - operating system plus installed applications publicly available. GNU/Linux, being the only free and open source operating system, is just a tool to do this. Success is, therefore, measured in terms of viability or practicability, as well as being able to help others, and not so much in terms of the overall number of users, or general adoption.

\section{References}

Arjona Reina, L. (2012) Translations in Libre Software. Madrid: Master Thesis, Universidad Rey Juan Carlos.

Baldwin, A. (2008) Linguas OS - Linux for Translators: a Review of Linguas OS. Available at: https://www.translatorscafe.com/cafe/EN/article85.htm [Accessed 20 June 2015].

Cronin, M. (2003) Translation and Globalization. London: Routledge.

Cronin, M. (2010) The Translation Crowd. Revista Tradumàtica 08/2010, 1-7. Available at: http://www.fti.uab.es/tradumatica/revista/num8/articles/04/04.pdf [Accessed 20 June 2015].

Diaz Fouces, O. (2010) Faça você mesmo: GNU/Linux para tradutores (softwares livres e tradução) Conferencia invitada promovida polo Departamento de Letras Modernas e o Centro Interdepartamental de Tradução e Terminologia da Faculdade de Filosofia, Letras e Ciências Humanas da Universidade de São Paulo. 
Diaz Fouces, O. (2011) ¿Merece la pena introducir el software libre en la formación de traductores profesionales? In Universitat de Vic (ed.) Anais das XI Jornadas de Traducción y Lenguas Aplicadas - Congreso Internacional "Didáctica de las lenguas y la traducción en la enseñanza presencial y a distancia" CDROM Language and Translation Teaching in FacetoFace and Distance Learning. Facultat de Ciències Humanes, Traducció i Documentació de la Universitat de Vic.

Diaz Fouces, O. and García González, M. (eds.) (2008) Traducir con software libre. Granada: Comares.

DistroWatch.com (n.d.) Distrowatch.com - Put the fun back into computing. Use Linux, BSD. Available at: http://distrowatch.com [Accessed 20 June 2015].

Dotsub (n.d.) dotsub - The leading source for video translation and captions. Available at: https://dotsub.com/about [Accessed 20 June 2015].

EU Commission (2015) Open Source Software Strategy 2014-2017. Available at: http://ec.europa.eu/dgs/informatics/oss_tech/strategy/strategy_en.htm [Accessed 20 June 2015].

EU Commission (2015b) Joinup - Share and reuse interoperability solutions for public administrations. Available at: http://joinup.ec.europa.eu [Accessed 20 June 2015].

FLISoL (n.d.) Festival Latinoamericano de Instalación de Software Libre. Available at: http://www.flisol.info [Accessed 20 June 2015].

FOSS4Trans (n.d.) A Catalogue of Free/Open-Source Software for Translators. Available at: http://traduccionymundolibre.com [Accessed 20 June 2015].

FSF (n.d.) Free Software Foundation. Available at: http://www.fsf.org/ [Accessed 20 June 2015].

FSFE (n.d.) Free Software Foundation Europe: Free Software and Education. Available at: http://fsfe.org/freesoftware/education/education.en.html [Accessed 20 June 2015].

García González, M. (2013) Free and Open Source Software in Translator Education. The MINTRAD Project In The International Journal for Translation \& Interpreting Research. 5-2, 125-148.

GETLT (n.d.) Grupo de Estudos das Tecnoloxías Libres da Tradución. Available at: http://webs.uvigo.es/getlt/ [Accessed 20 June 2015].

GTK (n.d.) The Google Translator Toolkit. Available at: https://translate.google.com/toolkit/ [Accessed 20 June 2015].

Hartley, Matt (2015) Secret to Desktop Linux Adoption, on-line page 2. Available at: http://www.datamation.com/open-source/secret-to-desktop-linux-adoption-2.html [Accessed 20 June 2015].

LinguasOS discussion group (n.d.) Available at: http://tech.groups.yahoo.com/ group/linguasos/ [Accessed 20 June 2015].

Linguee (n.d.) English-German Dictionary. Available at: http://www.linguee.com [Accessed 20 June 2015].

Linux Counter Project (n.d.) Get counted as a Linux User. Available at: https://www.linuxcounter.net [Accessed 20 June 2015].

Linux Foundation (2015) The Linux Foundation. Available at: http://www.linuxfoundation. org/ [Accessed 20 June 2015]. 
Matecat (n.d.) Welcome to MateCat! Post-editing and outsourcing made easy. Available at: https://www.matecat.com/features/ [Accessed 20 June 2015].

Mcllroy, M. D., Pinson, E. N. and Tague, B. A. (1978) Unix Time-Sharing System Forward In The Bell System Technical Journal, 1978, 57 (6, part 2). Bell Laboratories, 1902.

MyMemory (n.d.) at translated.net. Available at: http://mymemory.translated.net [Accessed 20 June 2015].

Net Market Share (n.d.) Desktop Operating System Market Share. Available at: http://www.netmarketshare.com/operating-system-market-share.aspx?

qprid=8\&qpcustomd=0\&qpsp=2015\&qpnp=1\&qptimeframe=Y [Accessed June 2015]

Prior, M. (2003) Close Windows, Open Doors in Translation Journal 7(1). Available at: accurapid.com/journal/23linux.htm [Accessed 20 June 2015].

Prior, M. (2010) Linux for Translators. Available at: http://www.linuxfortranslators.org [Accessed 20 June 2015].

Sandrini, P. (2012) Translationstechnologie im Curriculum der Übersetzerausbildung In Zybatow, L. and Małgorzewicz, A. (eds.) Sprachenvielfalt in der EU und Translation. Translationstheorie trifft Translationspraxis. Neisse, 107-120. Available at: http://www2.uibk.ac.at/downloads/trans/publik/transtech-wroclaw.pdf [Accessed 20 June 2015].

Stallman, R. (2014) Linux and the GNU System. Available at: http://www.gnu.org/gnu/linuxand-gnu.en.html [Accessed 20 June 2015].

The Linux for Translators Forum (2002) Available at: https://groups.yahoo.com/neo/ groups/linuxfortranslators/info [Accessed 20 June 2015].

The Rosetta Foundation (n.d.) Trommons. Available at: http://www.therosettafoundation.org/trommons/ [Accessed 20 June 2015].

tuxtrans discussion group (n.d.) Available at: http://groups.google.de/group/tuxtrans/ [Accessed 20 June 2015].

tuxtrans (n.d.) tuxtrans - GNU/Linux Desktop for Translators. Available at: http://www.tuxtrans.org [Accessed 20 June 2015].

Unicode website (n.d.) The Unicode Consortium. Available at: http://unicode.org/ [Accessed 20 June 2015].

W3Schools (n.d.) OS Platform Statistics and Trends. Available at: http://www.w3schools.com/ browsers/browsers_os.asp [Accessed 20 June 2015].

Wikipedia (n.d.) Usage share of operating systems. Available at: https://en.wikipedia.org/ wiki/Usage_share_of_operating_systems [Accessed 20 June 2015].

Zetzsche, Jost (2014) The Translator's Toolbox. A Computer Primer for Translators. International Writers' Group, LLC. 
